\title{
Astronomical and space-based systems engineering
}

\author{
Benoît Mosser ${ }^{1}$ and Alain Abergel ${ }^{2}$ \\ ${ }^{1}$ LESIA, Observatoire de Paris, \\ CNRS, Université Pierre et Marie Curie, Université Denis Diderot, Observatoire de Paris, \\ 92195 Meudon, France \\ email: benoit.mosser@obspm.fr \\ ${ }^{2}$ Institut d'Astrophysique Spatiale, \\ UMR8617, Université Paris XI, Bâtiment 121 \\ 91405 Orsay Cedex, France \\ email: alain.abergel@ias.u-psud.fr
}

\begin{abstract}
The Master's degree "Outils et Systèmes de l'Astronomie et de l'Espace" (OSAE, "Astronomical and Space-based Systems Engineering") is intended for students interested in Astronomy and Space technology. Students undergo a comprehensive training in partnership with international-level laboratories and with leading private companies. The degree provides physicists with a wide range of skills, appropriate for those whose intention is to participate in subsystems, equipment and engineering systems, and also for future project managers, working in the aerospace industry or similar technological industries or in national and European agencies. The 1-year course is given in collaboration with national and international institutions, laboratories and industries. It includes an extended training period (5 to 6 months) and a theoretical and practical specialization given by university and industrial teachers. It benefits from the network of laboratories associated with the Astronomy and Astrophysics doctorate school of the Ille-de-France.
\end{abstract}

Keywords. Master's degree, Space, System, Engineering, Instrumentation, Numerical techniques

\section{Introduction}

The techniques used today for astronomy and space projects are complex systems pushed to the highest performance levels. They are conceived in research laboratories with international support, carried out with strong industrial partnerships and executed by national and European agencies, e.g. the French National Centre for Space Studies (CNES), the European Space Agency (ESA) and the European Southern Observatory (ESO). The conception and the operation of these systems, together with contacts between engineers, researchers and industrialists, offers exceptional training possibilities in a high-technology and advanced international context (Fig. 1).

The Master's degree "Outils et Systèmes de l'Astronomie et de l'Espace" (OSAE, "Astronomical and Space-based Systems Engineering") is intended for students in physics, applied physics, technology, electronics, computer science, materials science etc., interested in astronomy and science or space technology. This short course (1 year) is given in collaboration with national and international institutions, laboratories and industries. It includes an extended training period ( 5 to 6 months) and a theoretical and practical specialization given by university and industrial teachers. The full description of the educational resources is given in Section 2. 
The Master's degree is associated with the doctorate school in Astronomy and Astrophysics. About 30 laboratories (CNRS, CEA, the Paris Observatory, Universities, ONERA) in the Ile-de-France region, as well as national and international institutions (CNES, IRAM, ESO, ESA/ESTEC) participate in this school; in addition, the degree Astronomical and Space-based Systems Engineering includes private industry. Students are presented with a complete training in partnership with international-level public and private laboratories. The rôles of each partner are elaborated in Section 3.

The degree trains physicists for a wide range of skills, appropriate for those whose intention is to take responsibility for subsystems, equipment and engineering systems, and also for future project managers, working in the aerospace industry or similar technology industries or in national and European agencies. Specialized industries, equipment producers, small subcontracting firms and laboratories from the public sector, are also possible outlets. Skills and jobs are described in Section 4.

Finally, in Section 5, we summarise our experience.

\section{Educational resources}

\subsection{Organisation}

The 1-year course is divided into three parts: the core syllabus and the specialization in Semester 1 and the training period in Semester 2. The core syllabus and the specialization include courses, practical work and projects. The specialization "computational physics" is directed towards digital techniques, programming tools, signal processing and simulations, the other specialization "instrumental techniques" focusses on the methods for pushing instrumentation to the highest performance levels in the constrained environments of large telescopes and space missions. A training in the conception of complex systems for space or ground-based instruments is delivered in the core syllabus.

In addition, students lead and develop practical projects. All together, the different modules introduce the many subjects necessary first to observe an astronomical object, than to process the data (Fig. 2).

Table 1 describes the resources of the modules taught in Semester 1.

- The "computational physics" specialization includes advanced programming tech-
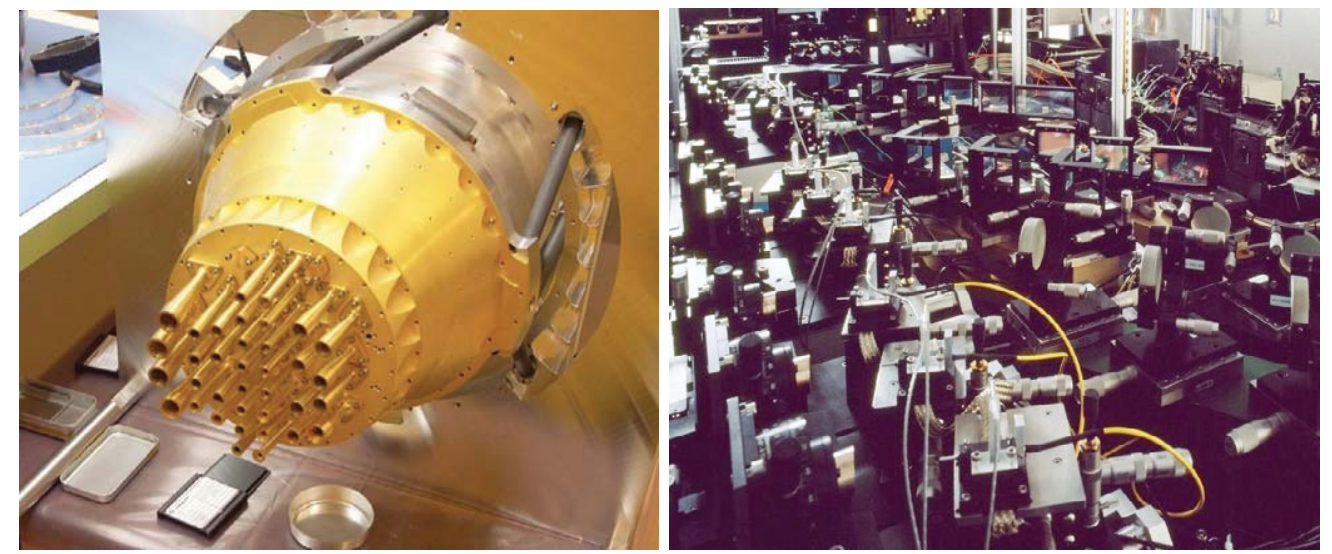

Figure 1. (Left) High Frequency Instrument (HFI) for the focal plane of the ESA Planck mission; (Right) Amber interferometer at ESO/VLTI. The goal of the Master's degree "Astronomical and Space-based Systems Engineering" is to train physicists whose intention is to take responsibility for the conception, the development, the operations and the exploitation of such instruments. 
Table 1. First semester

\begin{tabular}{lll}
\hline \multicolumn{1}{c}{ Core syllabus } & \multicolumn{2}{c}{ Specializations } \\
\hline & Instrumental techniques & Computational physics \\
\hline - Signal detection & - Guided and active optics & - Digital processing \\
- Data processing, automation & - Microwaves, radars and ap- & - Structure calculations (me- \\
& plications & chanical, vibration) \\
- Structure calculation & - Sensors, microelectronics & - Data acquisition and process- \\
& ing \\
- Systems and projects & - Thermical, cryogenics & - Filtering. Wavelet transform \\
- Industry & - Space environment & - Object oriented programming \\
- Practical work in instrumen- & & - Electronics and programming \\
tation & & onboard \\
- Computing science & & \\
- Astronomy & & \\
- English language & & \\
- Project & & \\
\hline
\end{tabular}

niques, civil engineering software, user interfaces, a complete training in the use of software, data acquisition and processing.

- The "instrumental techniques" specialization includes training in signal detection (sensors, optics, and electronics) under very demanding conditions for space projects or large observatories.

- During the 5 to 6 -month long training period, students work in industry, space agencies, foreign laboratories or observatories (Table 2).

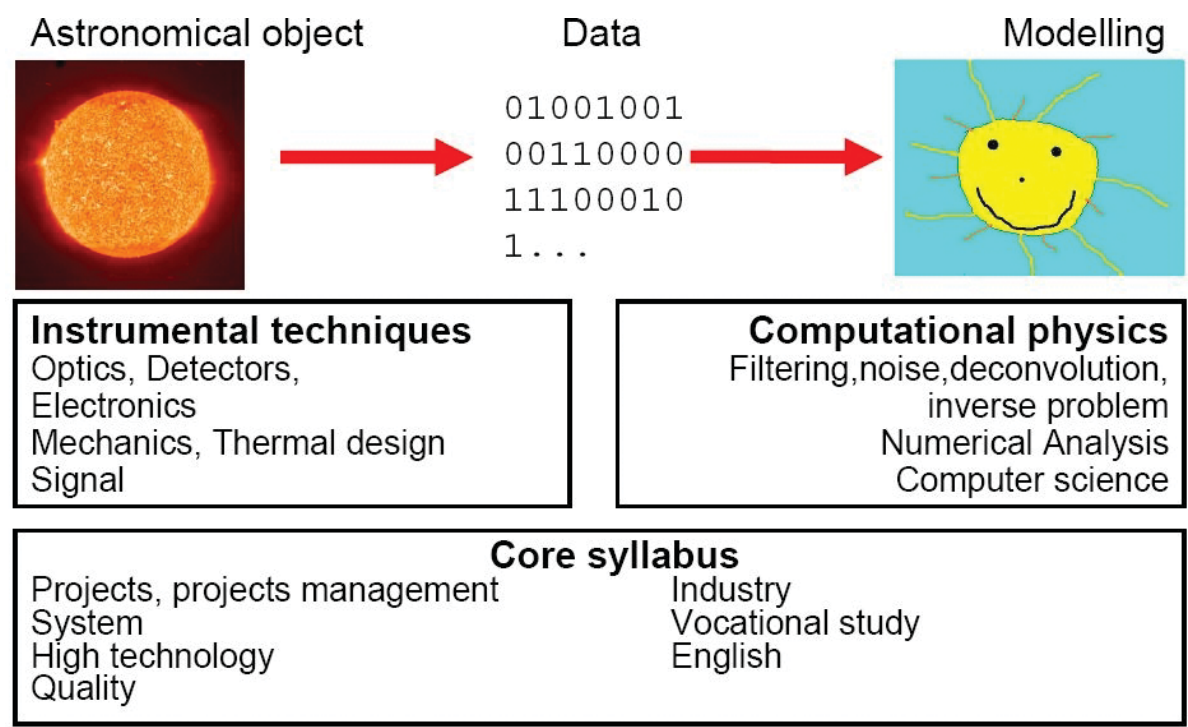

Figure 2. The philosophy of the Master OSAE: the different modules give a detailed view on each step necessary to observe an astronomical objet (specialization in instrumental techniques) and to process the data (specialization in data processing). All of these steps developed in detail during each specialization period are first introduced during the core syllabus. 
Table 2. Second semester: training period

Examples of training periods in industry

- Setup of an optical bench EADS, Toulouse

- Radiometric test bench for APS and CDD de- SODERN

France tectors

- Modeling and design of a deformable mirror

REOSC/SAGEM

France

- Characterization of a C-MOS detector

- New technology mirrors

EADS, Toulouse France

- Calibration of an infrared detector

Imagine Optics, Or- France say

$\ldots$

Alcatel, Cannes France

Examples of training periods in

space agency, foreign laboratory or observatory

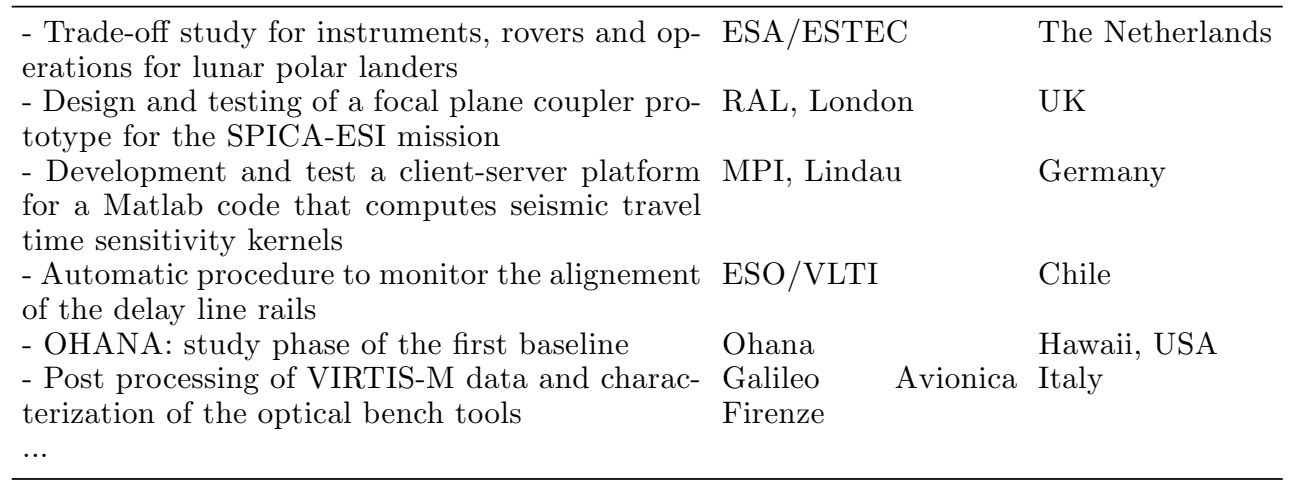

\subsection{Specific lectures}

Lectures specific to the Master's define its spirit. They are all based on special skills of the laboratories involved in the Master's, including space-borne but also ground-based

Table 3. Fact sheet

\begin{tabular}{ll}
\hline Opening & 2000 \\
\hline Core syllabus & $380 \mathrm{hr}$ \\
Specialization & $150 \mathrm{hr}$ \\
Training course & $5-6 \mathrm{months}$ \\
\hline Candidates per session & $\simeq 18$ \\
- withdrawals before the examination & $3 \%$ \\
- success at the exam & $98 \%$ \\
\hline Education completed with a PhD in instrumental & $15 \%$ \\
or numerical engineering & \\
\hline Placement rate as engineer & $\simeq 85 \%$ \\
Median time before placement & 2 months \\
Median time before a rolling contract & 2.5 years \\
\hline Engineer in the industry & $62 \%$ \\
Engineer in agencies, laboratories or observatories & $38 \%$ \\
\hline Web site (in French + page in English) & http://osae.obspm.fr/ \\
Web site of the association of the OSAE students & http://ww. osae.fr/ \\
\hline
\end{tabular}

Values averaged over 7 groups of students, having graduated in the years 2001-2007. 
projects. We manage to introduce multiple aspects in each lecture: for example, adaptive optics gives the opportunity to study in detail optics, mechanics, automation and systems.

- The "System and space project" module presents the specific characteristics of the organization of space projets. It introduces the development in different phases, as well as the concept of a system, the systems approach, the functional analysis techniques.

- The "Object oriented programming" module is proposed as a project for the modeling of a complex system (e.g. the detection chain of adaptive optics). The module is organized in a collaborative environment: each sub-group is in charge of a part of a subsystem; a student acts as a project manager.

- The "Project study" is a personal contribution to a Phase 0 or Phase A project. With this module, students undergo a detailed analysis of a subsystem selected among the critical subsystems of the project.

- The training period, in industry, space agency, foreign laboratory or observatory is oriented towards system approach (Table 2). Long duration is profitable for efficient contributions.

- A 2-day visit of a space technology center (ESTEC, Fig. 3) or a large ground-based facility (Nançay Radio Telescope) gives the opportunity to discover the facilities for the integration and tests of space-borne or ground-based projects.

Further informations on the degree are given in Table 3.

\section{Partners}

The degree benefits from the experience and skills of the partners which are involved in the most recent and largest projects in astronomy (e.g., CoRoT, Planck, Herschel, Mars Express, Rosetta, JWST, Bepi Colombo, NAOS/VLT, OHANA, VISIR/VLT, ...). Due

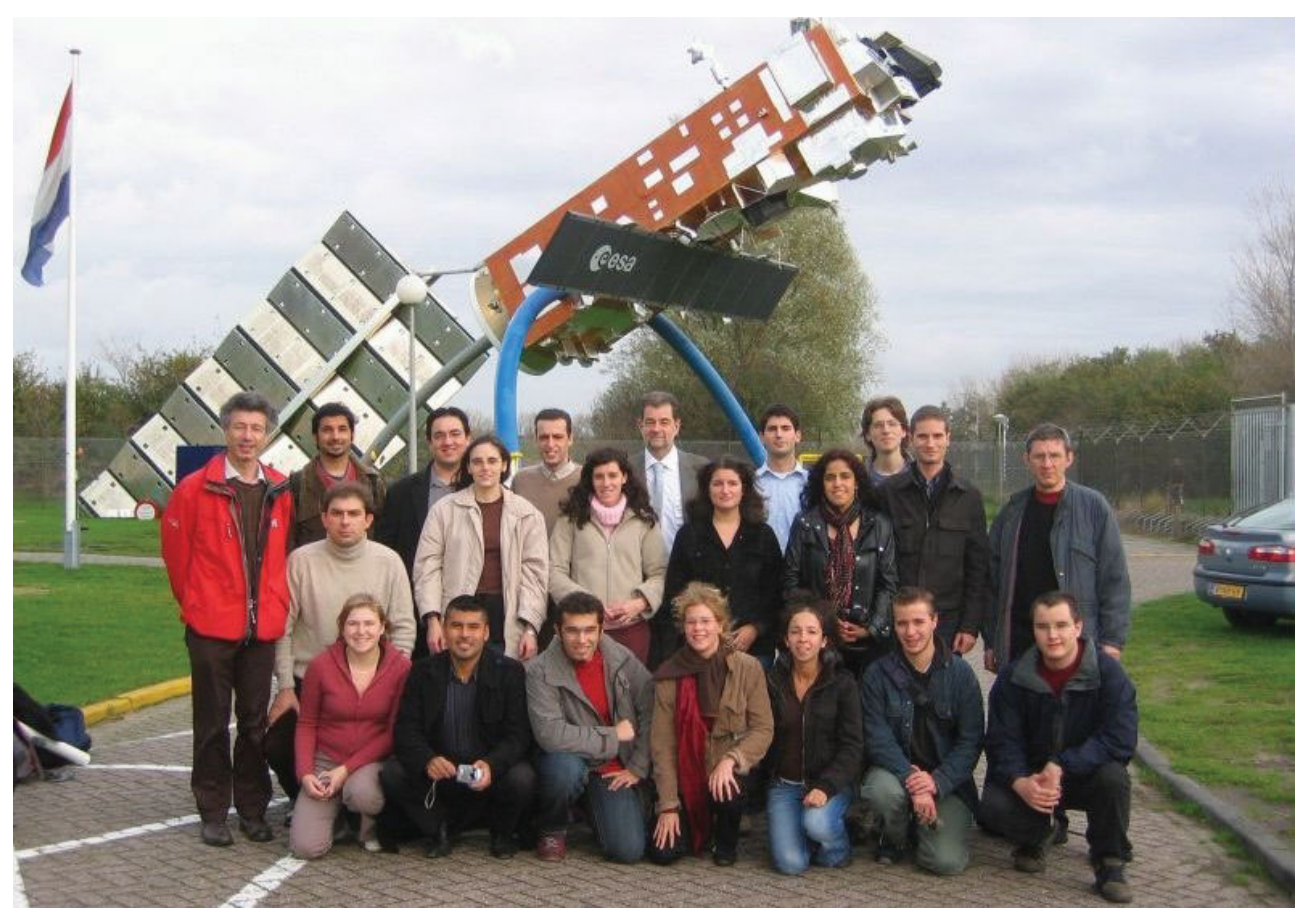

Figure 3. OSAE students visiting ESTEC, the European space research and technology centre. 
Table 4. Main partners

Universities supporting the Master's

Observatoire de Paris

Université Pierre et Marie Curie, Paris 6

Université Denis Diderot, Paris 7

Université Paris-Sud, Paris 11

Meudon
Paris
Paris
Orsay

Meudon

Paris

Orsay

Space laboratories involved in the Master's ${ }^{b}$

IAS, Institut d'Astrophysique Spatiale, Univer- Orsay sité Paris-Sud

LESIA, Laboratoire d'Études Spatiales et Meudon

France

d'Instrumentation en Astrophysique, Paris Observatory

LPP, Laboratoire de Physique des Particules, Palaiseau

École Polytechnique

SAp, Service d'Astrophysique, CEA

Saclay

II

"1

"I

Observatories, National and International Agencies*

\begin{tabular}{|c|c|c|}
\hline CFHT & Mauna-Kea & Hawaii \\
\hline CNES, Centre National d'Études Spatiales & Toulouse & France \\
\hline $\begin{array}{l}\text { ESA/ESTEC, European Space Research and } \\
\text { Technology Centre }\end{array}$ & Nordwijk & The Netherlands \\
\hline ESO, European Southern Observatory & Garching & Germany \\
\hline $\begin{array}{l}\text { IRAM, Institut de Radio-Astronomie Mil- } \\
\text { limétrique }\end{array}$ & Grenoble & France \\
\hline $\begin{array}{l}\text { ONERA, Office National d'Études et de } \\
\text { Recherche en Aéronautique }\end{array}$ & Chatillon & France \\
\hline
\end{tabular}

\begin{tabular}{lll}
\hline & Industry* $^{*}$ & \\
\hline AER & Chevilly-Larue & France \\
Astrium satellites & Toulouse & France \\
Carlo Gavazi Space & Padova & Italy \\
CILAS & Orléans & France \\
EADS Space transportation & Les Mureaux & France \\
Imagine Optics & Orsay & France \\
SAGEM/REOSC & Saint-Pierre du Perray & France \\
SESO & Aix-en-Provence & France \\
SODERN / EADS & Limeil-Brevannes & France \\
Thalès Alenia Space & Cannes & France \\
$\ldots$ & & \\
\hline
\end{tabular}

${ }^{b}$ Major contribution in bold font.

${ }^{*}$ Partners involved via teaching or training.

to the nature of these projects, the partnership brings together laboratories, agencies and industrialists (Table 3). The rôle of the partners are, depending on the case, similar or complementary. Research and development are conducted everywhere. Phase 0 work occurs mainly in the laboratories; phase A work too, in close interaction with the agencies. Further phases are supervised by the agencies, with most of the realizations by the industry, but for very unusual devices realized in the laboratories. External experts from industry and agencies participate in the modules. Trainings are favored in the agencies and industries, while a large fraction of them, about one third, occur in an international context (Table 2). 
Table 5. Job types of OSAE graduates

\begin{tabular}{ll}
\hline Research \& Development Engineer & Project Controller \\
System Engineer & Cost Engineer \\
Project Engineer & Mechanical Engineer \\
Product Assurance \& Quality Engineer & Thermal Engineer \\
Test Engineer & Applications Engineer \\
Qualification Engineer & Software Engineer \\
Safety Engineer & \\
\hline
\end{tabular}

The complex interactions between the actors of space and astrophysics have consequences for the careers of the graduates, as is explained in the following Section.

\section{Skills and jobs}

The objective of the Master's degree, intended to prepare for system engineering, are well met since $85 \%$ of the jobs occupied by the graduates of the Master's OSAE are related to engineering in the high-technology domain, in industry, space agencies, space laboratories or observatories. The importance of the rôle of agencies and observatories in the astrophysical and space sector is apparent in the large fraction of graduates working there, about one third (Table 3). A non-negligible fraction of the graduates, about $15 \%$, complete the Master's education with a $\mathrm{PhD}$ in instrumental or computational engineering.

The degree, given in an astrophysical context, applies to all high-technology fields, especially when quality plays an important rôle, or when complex systems are involved. The types of most of the jobs occupied by the graduate students are given in Table 5 .

Discussions with the graduates and with their employers give the reasons explaining the successful placement of the graduates:

- The Master delivers a multiple set of skills in instrumentation, digital processing and information technology.

- Emphasizing the systems approach yields a highly valued skill.

- Quality and high-technology are naturally associated with space-borne and groundbased projects in astronomy, thus students can apply successfully for jobs in quality and high-technology.

\section{Discussion}

The 9-year experience of the Master's degree can be summarized by a few statements:

- In France, education of engineers is most often given in Engineer Schools and not at the University. The success of the placement of our students as engineers, hence the success of the degree, shows that the knowhow and skills of the astronomical laboratories and of their partners yields a high performance education. It emphasizes too, if necessary, that astronomy is not only beautiful galaxies and stars, but also a science which stimulates the development of extremely complex systems that works successfully.

- Projects and collaboration between engineers, researchers and industrialists translate easily into a degree where engineers, researchers and industrialists collaborate. This gives a degree allowing a smooth and successful transition from the University to the employment market.

- Training possibilities in astronomy are able to propose exceptional experience in a high-technology and advanced international context. Having participated, for example, 
in the test of the alignment and setup of the VLTI delay lines is an invaluable experience that will fully benefit the CV of a young professional.

- Key science in astrophysics comes largely from key projects. Most of the important characteristics of these projects translates into very generic keywords: research and development, complex system, project organization, quality. Early career engineers having benefitted from such an education can apply these skills in all high-technology domains.

\section{Acknowledgements}

Being in charge of the Master OSAE is for us a real pleasure, and we warmly thank all of the engineers, professors and researchers involved in this Master's degree. The foresight of many persons allowed its creation in 2000, among whom we should cite Michel Combes, astronomer and former president of the Paris Observatory, Pierre Encrenaz, professor at the Pierre-et-Marie-Curie University (Paris 6) and Pierre Léna, professor at the DenisDiderot University (Paris 7). The Master's degree benefitted from the support of many other people: we apologise for not mentioning all of them, but we must single out Sonia Akrour for her invaluable rôle presence as the Master's secretariat and administration. 